\title{
Physics at the B Factories
}

\author{
T.E. Browder, University of Hawaii at Manoa
}

(To appear in the Proceedings of From the Smallest to the Largest Distances, a conference in honor of Tranh Thanh Van in Moscow, Russia).

\begin{abstract}
We review recent progress at the two $e^{+} e^{-} \mathrm{B}$ factories. The first measurement of $\mathrm{CP}$ violation and the prospects for measuring all the angles of the unitarity triangle are discussed.
\end{abstract}

\section{High Luminosity $e^{+} e^{-} \mathbf{B}$ factories}

\section{$1.1 \quad$ Introduction}

Many important discoveries in high energy physics were announced in the Rencontres de Moriond series. For example, at the 1987 Rencontres de Moriond in a presentation by $\mathrm{H}$. Schroeder, the ARGUS collaboration described the surprising discovery of large $B_{d}-\bar{B}_{d}$ mixing [1]. The implications of this observation were important for the experimental program on $\mathrm{CP}$ violation. As a result, the theoretical proposal of Bigi, Carter and Sanda 2] that there would be large CP asymmetries in hadronic $B$ decays to $\mathrm{CP}$ eigenstates became experimentally feasible. This initiated the program which would eventually lead to the plans for the high luminosity $e^{+} e^{-}$B factories.

Two international high luminosity $e^{+} e^{-} B$ factories have now been completed and are collecting data in the United States (PEPII/BABAR) and Japan (KEKB/BELLE) [3], [4]. The goal of these experiments is to measure $\mathrm{CP}$ asymmetries in the $B$ system and test the foundations of the Standard Model. 
The accelerators were commissioned with remarkable speed starting in late 1998. The experiments starting physics data taking in 1999. By the time of the XXXVI Rencontres de Moriond in winter 2001, there were hints (at the $1.7 \sigma$ level) of $\mathrm{CP}$ violation from both BABAR and BELLE. In the summer of 2001, these two experiments announced the observation of the first statistically significant signals for CP violation outside of the kaon system.

\subsection{CP Violation Induced by Mixing}

In 1973, Kobayashi and Maskawa (KM) first proposed a model where $C P$ violation is incorporated as an irreducible complex phase in the weak-interaction quark mixing matrix [5]. The idea, which was presented at a time when only the $u, d$ and $s$ quarks were known to exist, was remarkable because it required the existence of six quarks. The subsequent discoveries of the $c, b$ and $t$ quarks, and the compatibility of the model with the $C P$ violation observed in the neutral $K$ meson system led to the incorporation of the KM mechanism into the Standard Model, even though it had not been conclusively tested experimentally.

The unitarity of the CKM matrix implies that the existence of three measurable phases. In the "Nihongo" convention, these are denoted

$$
\phi_{1} \equiv \arg \left(-\frac{V_{c d} V_{c b}^{*}}{V_{t d} V_{t b}^{*}}\right), \quad \phi_{2} \equiv \arg \left(-\frac{V_{u d} V_{u b}^{*}}{V_{t d} V_{t b}^{*}}\right), \quad \phi_{3} \equiv \arg \left(-\frac{V_{c d} V_{c b}^{*}}{V_{u d} V_{u b}^{*}}\right) .
$$

while at SLAC these angles are usually referred to as $\beta, \alpha$ and $\gamma$, respectively.

A non-zero value of $\phi_{C P}$ results in the time dependent asymmetry

$$
A_{f}=\frac{R\left(B^{0} \rightarrow f_{C P}\right)-R\left(\bar{B}^{0} \rightarrow f_{C P}\right)}{R\left(B^{0} \rightarrow f_{C P}\right)+R\left(\bar{B}^{0} \rightarrow f_{C P}\right)}=\xi_{f} \sin 2 \phi_{C P} \cdot \sin \left(\Delta m \cdot\left(t_{2} \pm t_{1}\right)\right),
$$

where $\xi_{f}$ is the $\mathrm{CP}$ eigenvalue $( \pm 1), \Delta m$ denotes the mass difference between the two $B^{0}$ mass eigenstates and $t_{1}$ and $t_{2}$ are the proper time for the tagged$B$ and $C P$ eigenstate decays, respectively. The + sign corresponds to the case where the $B^{0}$ and $\overline{B^{0}}$ are in an even L orbital angular momentum state, the - sign obtains for odd L states. A determination of $A_{f}$ thus provides a measurement of $\sin 2 \phi_{C P}$.

We note that due to the restrictions of quantum mechanics, time integrated asymmetries at the $\Upsilon(4 S)$ resonance (which corresponds to the - sign

in the above equation) are identically zero. Therefore, one must make time 
dependent measurements. Since the pairs of B mesons are produced nearly at rest in the usual arrangement at threshold, the $\Upsilon(4 S)$ center of mass frame must be boosted. This is accomplished by the use of beams with asymmetric energies. For example at KEK-B, $\beta \gamma \sim 0.43$, (at PEP-II, $\beta \gamma \sim 0.56$ ) and the typical $B$ meson decay length is dilated from $\sim 20 \mu \mathrm{m}$ to $\sim 200 \mu \mathrm{m}$, which is measurable with solid-state vertex detectors close to the interaction point. At the symmetric CESR storage ring which uses equal energy beams, these measurements are not possible.

\subsection{Accelerators and Detectors}

The $e^{+} e^{-} B$ factory experiments require substantial improvements in accelerator technology to achieve luminosities in excess of $3 \times 10^{33} / \mathrm{cm}^{2} / \mathrm{sec}$ and data samples with integrated luminosities of $\mathrm{O}\left(100 \mathrm{fb}^{-1}\right)$ as well as some improvements in detector technology. The properties of the B factory accelerators are summarized in Table 1 . The properties of the individual bunches are not dramatically different from what had been achieved at conventional storage rings such as CESR. Instead to obtain high luminosity, the asymmetric $\mathrm{B}$ factories use double storage rings and very large numbers of bunches. This is a domain of accelerator operation which had not been fully explored previously and to avoid instabilities involving coupling of bunches requires the operation of complex feedback systems.

To minimize parastic collisions between incoming and outgoing bunches, KEK-B chose the crossing angle approach while SLAC takes advantage of the energy asymmetry and uses magnetic separation to achieve this separation. A small crossing angle of $\pm 2.1 \mathrm{mrad}$ had been successfully used at CESR to achieve high luminosity. KEK-B employs a somewhat larger \pm 11 mrad crossing angle. There is still some worry that coupled oscillations of the longitudinal and transverse degrees of the beam (synchro-betatron oscillations) may be excited, so KEK-B continues $\mathrm{R}+\mathrm{D}$ to develop special $\mathrm{RF}$ cavities to rotate the bunches so that they collide head on at the interaction point. The magnetic separation approach gives rise to somewhat larger experimental backgrounds and imposes tight constraints on the engineering of the interaction region.

The performance achieved by both accelerator complexes in a very short time is quite remarkable. Both started physics running in 1999. By the end of the summer of 2001, PEP-II had integrated about $40 \mathrm{fb}^{-1}$ while KEK-B had 
integrated $33 \mathrm{fb}^{-1}$. The peak luminosity for PEP-II was $3.9 \times 10^{33} / \mathrm{cm}^{2} / \mathrm{sec}$ while KEK-B had achieved $4.5 \times 10^{33} / \mathrm{cm}^{2} / \mathrm{sec}$. In the case of KEK-B, the beam currents are still far below the design values. (Also note that the operating parameters currently used are constantly changing and somewhat different from those in the design (Table 1)).

The experimental measurements of $\mathrm{CP}$ violation require tagging of the $B$ flavor, measurement of the time dependence of the decay and high momentum particle identification. As shown in Table 2, in contrast to the accelerators, the detectors are quite similar with the exception of the particle identification systems [3], [4].

\begin{tabular}{|c|c|c|c|c|c|}
\hline \multicolumn{4}{|c|}{\begin{tabular}{l|c} 
Table 1: Design Parameters for B-Factor \\
\end{tabular}} & \multicolumn{2}{|c|}{ SLAC } \\
\hline & & LER & HER & LER & HER \\
\hline Energy & $E(\mathrm{GeV})$ & 3.5 & 8.0 & 3.1 & 9.0 \\
\hline Luminosity & $L\left(\mathrm{~cm}^{-2} \mathrm{~s}^{-1}\right)$ & \multirow{2}{*}{\multicolumn{2}{|c|}{$\begin{aligned} & 1 \times 10^{34} \\
\pm & 11 m r(\mathrm{crab})\end{aligned}$}} & \multicolumn{2}{|c|}{$3 \times 10^{33}$} \\
\hline Collision mode & & & & \multicolumn{2}{|c|}{ Head-on } \\
\hline Circumference & $C(\mathrm{~m})$ & \multicolumn{2}{|c|}{3018} & \multicolumn{2}{|c|}{2199} \\
\hline Beta Function & $\beta_{x}^{*} / \beta_{y}^{*}(\mathrm{~cm})$ & $100 / 1$ & $100 / 1$ & $37.5 / 1.5$ & $75 / 3$ \\
\hline Tune shift & $\xi_{x} / \xi_{y}$ & \multicolumn{2}{|c|}{$0.05 / 0.05$} & \multicolumn{2}{|c|}{$0.03 / 0.03$} \\
\hline Emittance & $\epsilon_{x} / \epsilon_{y}(\mathrm{~nm})$ & $19 / 0.19$ & $19 / 0.19$ & $64 / 2.6$ & $48.2 / 1.9$ \\
\hline Energy spread & $\sigma_{E} / E\left(10^{-4}\right)$ & 7.7 & 7.2 & 9.5 & 6.1 \\
\hline Total Current & $I(\mathrm{~A})$ & 2.6 & 1.1 & 2.14 & 0.98 \\
\hline No. of Bunches & $N_{B}$ & \multicolumn{2}{|c|}{5120} & \multicolumn{2}{|c|}{1658} \\
\hline Bunch spacing & $S_{B}(\mathrm{~m})$ & \multicolumn{2}{|c|}{0.6} & \multicolumn{2}{|c|}{1.26} \\
\hline RF frequency & $f_{R F}(\mathrm{MHz})$ & \multicolumn{2}{|c|}{508} & \multicolumn{2}{|c|}{476} \\
\hline RF voltage & $V_{c}(\mathrm{MV})$ & 22 & 48 & 9.5 & 18.5 \\
\hline Cavity type & & ARES & super & 1-cell & ormal \\
\hline No. of Cavities & $N_{c}$ & 28 & 60 & 10 & 20 \\
\hline
\end{tabular}

The most challenging requirement for the detector is the detection of short lived vertices. The experiments at both $B$ factories use double-sided silicon strips to deal with this requirement. These have been used previously at ALEPH, DELPHI, and at CLEO II and III. Nevertheless, the implementation is still far from routine.

The other difficult requirement for the detector is the separation of kaons from pions. At high momentum, this is needed to distinguish $\bar{B}^{0} \rightarrow \pi^{+} \pi^{-}$ 
from $\bar{B}^{0} \rightarrow K^{-} \pi^{+}$. The former is a spectator $b \rightarrow u$ mode which is used to determine one of the angles in the unitarity triangle while the latter is an example of a gluonic penguin $b \rightarrow s g^{*}$ decay. Similarly, the experiments must distinguish the Cabibbo suppressed mode $B^{-} \rightarrow D^{0} K^{-}$from the much more abundant and kinematically similar Cabibbo favored mode $B^{-} \rightarrow D^{0} \pi^{-}$. At lower momenta, particle identification is essential for flavor tagging.

Two approaches for high momentum particle identification have been implemented. Both are based on the use of Cerenkov radiation. At BELLE, aerogel Cerenkov radiators are used. Blocks of aerogel are readout directly by fine-mesh phototubes which have high-gain and operate comfortably in a 1.5 Tesla magnetic field. Since the threshold for the aerogel is around $1.5 \mathrm{GeV}$, below this momentum $\mathrm{K} / \pi$ separation is carried out using high precision time-of-flight scintillators with resolution of 95 ps. The aerogel and TOF counter system are complemented by $\mathrm{dE} / \mathrm{dx}$ measurements in the central drift chamber. The $\mathrm{dE} / \mathrm{dx}$ system provides $\mathrm{K} / \pi$ separation around 2.5 $\mathrm{GeV}$ in the relativistic rise region and below $0.7 \mathrm{GeV}$. For high momentum kaons, an efficiency of $88 \%$ with a misidentification probability of $9 \%$ has been achieved.

At BABAR, Cerenkov light is produced in quartz bars and then transmitted by internal reflection outside of the detector through a water tank to a large array of phototubes where the ring is imaged. The detector is referred to by the acronym DIRC. It provides particle identification over the full momentum range for particles that are energetic enough to reach it. Additional particle identification is provided by $\mathrm{dE} / \mathrm{dx}$ measurements from the drift chamber and from the 5-layer silicon detector system.

For tracking, both B-factory experiments use conventional drift chambers with a low-Z helium based gas to minimize multiple scattering and SR (synchrotron radiation) backgrounds.

To detect photons and electrons, the $B$ factory detectors use large arrays of $\mathrm{CsI}(\mathrm{Tl})$ crystals located inside the coil of the magnet. These were used previously with great success by the CLEO II experiment. In BABAR and BELLE, another novel feature is a hadron calorimeter which is used for $K_{L}$ and muon detection and allows the use of $B^{0} \rightarrow \psi K_{L}$ in addition to the CP eigenstate $B \rightarrow \psi K_{s}$. 


\begin{tabular}{|l|c|c|}
\hline \multicolumn{3}{|c|}{ Table 2: B Factory Detectors } \\
\hline Name & BaBar & BELLE \\
Jazphant & Jazz $B$ \\
$\theta_{\text {crossing }}$ & $0 \mathrm{mr}$ & $\pm 11 \mathrm{mr}$ \\
Vertex & 5 layers & 3 layers \\
$r(\mathrm{~cm})$ & $3.2-14.4$ & $3-5.8$ \\
CDC & 40 layers & 50 layers \\
$r(\mathrm{~cm})$ & $24-80$ & $9-86$ \\
PID & DIRC+dE/dx & Aerogel/TOF $+\mathrm{dE} / \mathrm{dx}$ \\
EM Cal & CsI(Tl $)$ & CsI(Tl $)$ \\
Magnet & $1.5 \mathrm{~T}$ & $1.5 \mathrm{~T}$ \\
$K_{L} / \mu$ & RPC & RPC \\
& Linseed Oil & Glass \\
DAQ & Digital & Q-to-T into \\
& Pipeline & multihit TDC \\
\hline
\end{tabular}

\subsection{The First Measurements of CP Violation}

The measurement requires the reconstruction of $B^{0} \rightarrow f_{C P}$ decays, the determination of the $b$-flavor of the accompanying (tagging) $B$ meson, the measurement of $\Delta t$, and a fit of the expected $\Delta t$ distribution to the measured distribution using a likelihood method.

BELLE reconstructs $B^{0}$ decays to the following $C P$ eigenstates: $J / \psi K_{S}$, $\psi(2 S) K_{S}, \chi_{c 1} K_{S}, \eta_{c} K_{S}$ for $\xi_{f}=-1$ and $J / \psi K_{L}$ for $\xi_{f}=+1$. BABAR includes all these modes except for $\eta_{c} K_{s}$. The two classes $\left(\xi_{f}= \pm 1\right)$ should have CP asymmetries which are opposite in sign.

Both experiments also use $B^{0} \rightarrow J / \psi K^{* 0}$ decays where $K^{* 0} \rightarrow K_{S} \pi^{0}$. Here the final state is a mixture of even and odd CP. The CP content can, however, be determined from an angular analysis of other $\psi K^{*}$ decays. The 
$\mathrm{CP}$ odd fraction is found to be small (i.e. $19 \pm 4 \%(16 \pm 3.5)$ in the BELLE (BABAR) analysis).

The CP eigenstate event samples are shown in Figs. 11 and 2. The distributions of beam constrained mass are shown for the modes other than $\psi K_{L}$. For the latter, the distribution of $p_{B}^{*}$, the momentum of the $\mathrm{B}$ candidate in the CM frame is shown. In the fully reconstructed modes, BELLE finds 747 events over a background of 58.6. For the $\psi K_{L}$ sample, there are 569 over a background of 223. The event yields from BABAR are similar. They observe a total of 1230 events over background of 200 . It is clear that the $\mathrm{CP}$ eigenstate samples that are used for the $\mathrm{CP}$ violation measurements are large and clean.

To identify the flavor of the accompanying $B$ meson, leptons, kaons, charged slow pions from $D^{*} \rightarrow D^{0} \pi^{+}$decays, and energetic pions from twobody $B$ decay (e.g. $\bar{B}^{0} \rightarrow D^{*+} \pi^{+}$) are used. A likelihood based method is used by BELLE. BABAR first separately tags leptons and then kaons. If these methods fail or conflict, two neural nets are then used to identify the remaining tags. The figure of merit for flavor tagging is the effective efficiency, $\epsilon_{e f f}$, which is $\epsilon\left(1-2 w^{2}\right)$ summed over all tagging categories. BELLE's method gives $\epsilon_{\text {eff }}=0.270 \pm 0.008_{-0.009}^{+0.006}$ while BABAR finds $\epsilon_{e f f}=0.261 \pm 0.012$ for their method.

The probabilities of an incorrect flavor assignment are determined directly from the data using exclusively reconstructed, self-tagged $B^{0} \rightarrow D^{*-} \ell^{+} \nu$, $D^{(*)-} \pi^{+}, D^{*-} \rho^{+}$and $J / \psi K^{* 0}\left(K^{+} \pi^{-}\right)$decays. The values of $w_{l}$ are obtained from the amplitudes of the time-dependent $B^{0} \bar{B}^{0}$ mixing oscillations: $\left(N_{\mathrm{OF}}-N_{\mathrm{SF}}\right) /\left(N_{\mathrm{OF}}+N_{\mathrm{SF}}\right)=\left(1-2 w_{l}\right) \cos \left(\Delta m_{d} \Delta t\right)$. Here $N_{\mathrm{OF}}$ and $N_{\mathrm{SF}}$ are the numbers of opposite and same flavor events. These wrong-tagging rates determined from data are then used in the CP fit for BELLE. To incorporate this information in their analysis, BABAR performs a simultaneous fit to the flavor tagged and CP eigenstate data samples.

The $f_{C P}$ vertex is determined using lepton tracks from $J / \psi$ or $\psi(2 S)$ decays, or prompt tracks from $\eta_{c}$ decays. The $f_{\text {tag }}$ vertex is determined from well reconstructed tracks not assigned to $f_{C P}$. Tracks that form a $K_{S}$ are not used. For BELLE, the typical vertex-finding efficiency and vertex resolution $(\mathrm{rms})$ for $z_{C P}\left(z_{\mathrm{tag}}\right)$ are $92(91) \%$ and $75(140) \mu \mathrm{m}$, respectively. Note that the resolution on the tag side includes a large additional contribution from charm decay. For BABAR, the rms resolution in $\Delta z=z_{C P}-z_{\text {tag }}$ is $180 \mu \mathrm{m}$ and their vertexing efficiency is $97 \%$. Once the tag and CP eigenstate vertices 
are reconstructed, the proper time is calculated from $\Delta z / \gamma \beta$.

Examination of the time dependent distributions from the two experiments (Figs. 3, 1, 5.) shows clear indications of $\mathrm{CP}$ violation. The raw time distributions for the $B^{0}$ and $\bar{B}^{0}$ samples are indeed asymmetric. This is most striking in the BELLE figure. Thus, $\mathrm{CP}$ violation is visible even in the raw time distributions. The time dependent asymmetry distributions for the $\xi_{f}= \pm 1$ samples are indeed opposite as expected. Finally, the flavor tagged samples show no time dependent asymmetry.

To extract the values of the CP violating parameter, the two experiments perform unbinned maximum likelihood fits to the time distributions of the tagged and vertexed events. The fits take into account the effects of background, vertex resolutions and incorrect tagging.

In the summer of 2001, the first significant measurements of the CP violating parameter sin $2 \phi_{1}$ were obtained by BELLE and BABAR. BELLE finds

$$
\sin 2 \phi_{1}=0.99 \pm 0.14 \pm 0.06
$$

with a statistical significance of greater than six standard deviations [7]. BABAR finds

$$
\sin 2 \phi_{1}=0.59 \pm 0.14 \pm 0.05
$$

with a significance of 4.1 standard deviations [8]. The results are based on data samples of comparable size (31 million and 32 million $B \bar{B}$ pairs, respectively). As discussed above, the efficiencies and resolutions of the two experiments are also quite comparable. However, the two measurements are only marginally consistent. Larger data samples and additional more precise measurements will be required to fully reconcile these two results.

\section{Determination of the other angles}

Experimental work on the determination of the other angles $\phi_{2}$ and $\phi_{3}$ has just started. To obtain precise results that test the validity of the Standard Model, much larger data samples will be required. Below I briefly describe some of the possible approaches.

To measure $\phi_{2}$ (a.k.a $\alpha$ ), the two most promising approaches involve the use of the decay modes $B^{0} \rightarrow \pi^{-} \pi^{+}$and $B^{0} \rightarrow \rho^{ \pm} \pi^{\mp}$. The former is an example of $\mathrm{CP}$ eigenstate and is the most straightforward approach. 
The interference in this mode between the direct decay and the decay via mixing leads to a $\mathrm{CP}$ violating asymmetry as in the charmonium decay mode $B^{0} \rightarrow \psi K_{s}$.

However, there are several additional complications. The decay amplitude for $B^{0} \rightarrow \pi^{+} \pi^{-}$contains a contribution from a tree diagram $(b \rightarrow u \bar{u} d)$ as well as a Cabibbo suppressed penguin diagram $(b \rightarrow s \bar{u} s)$. The penguin contribution is not negligible and has a weak phase that is different from the phase of the larger tree amplitude, which is zero in the usual parameterization. Therefore the time dependent asymmetry, proportional to $\sin (\Delta m \Delta t)$, which is measured is not equal to $\sin 2 \phi_{2}$ but instead will have a large unknown correction. The presence of the extra contribution also induces an additional time dependent term proportional to $\cos (\Delta m \Delta t)$. This is called penguin pollution.

There are a number of other purely experimental complications. The branching fraction for the $B^{0} \rightarrow \pi^{+} \pi^{-}$decay is somewhat small. Using $22.6 \times 10^{6} B \bar{B}$ pairs BABAR finds $\mathcal{B}\left(B^{0} \rightarrow \pi^{+} \pi^{-}\right)=(4.1 \pm 1.0 \pm 0.7) \times 10^{-6}$ [9], while using a sample of $11.1 \times 10^{6} B \bar{B}$ pairs BELLE finds $\mathcal{B}\left(B^{0} \rightarrow\right.$ $\left.\pi^{+} \pi^{-}\right)=(5.6 \pm 2.3 \pm 0.4) \times 10^{-6}$ [10]. Moreover, even after the application of high momentum particle identification, the CP eigenstate signal sits on a large continuum background. The signal to continuum background ratio is a function of the tag method; this effect must be taken into account in the $\mathrm{CP}$ extraction. The conventional continuum suppression variables are also correlated with tagging. There is still residual background from misidentified $B^{0} \rightarrow K^{+} \pi^{-}$as well.

Despite these difficulties, the first attempts to determine $\sin 2 \phi_{2}$ using the $\pi^{+} \pi^{-}$mode have started. BABAR reports results of a preliminary fit which gives $\sin 2 \phi_{2}$ (eff) $=0.03_{-0.56}^{+0.53} \pm 0.11$. BELLE expects a sensitivity of \pm 0.6 and will present a result soon. The size of the correction for penguin pollution in $\sin 2 \phi_{2}(e f f)$ is unknown.

The angle $\phi_{3}$ is particularly difficult to measure. There are at least three methods which give some information on this angle.

The method which requires the least data but has the largest theoretical uncertainty involves the measurement of the branching fractions of the $K \pi$ and $\pi \pi$ decay modes. The underlying idea is to compare decays where the interference of the penguin and tree amplitudes are different to extract about information about the phase $\phi_{3}$. For example, if $\phi_{3}>90^{\circ}$, then the interference term is constructive for $B^{0} \rightarrow K^{+} \pi^{-}$and destructive for $B^{0} \rightarrow \pi^{+} \pi^{-}$, 
leading to a ratio for the widths of the two modes greater than one. Conversely, if $\phi_{3}<90^{0}$ then a $K^{-} \pi^{+} / \pi^{+} \pi^{-}$ratio less than unity is expected. The current data somewhat favor $\phi_{3}>90^{\circ}$ whereas the indirect CKM fits prefer $\phi_{3} \sim 60^{\circ}$. The interpretation of this discrepancy is still unclear.

A theoretical industry has been developed to extract the maximum possible information on $\phi_{3}$ from these and related decay modes 11]. However, it has been argued by some theorists that other smaller diagrams or final state interaction effects may invalidate these analyses [12], [13], [14].

Another method to determine $\phi_{3}$ uses the Cabibbo suppressed decay mode $B^{-} \rightarrow D^{0} K^{-} 15$. . In the case that $D^{0} \rightarrow f_{C P}$, where $f_{C P}$ is a CP eigenstate such as $K^{-} K^{+}, \pi^{+} \pi^{-}, K_{s} \pi^{0}$, two amplitudes with different weak phases will interfere. The asymmetry and rate again involve the phase of $V_{u b}$. This method is theoretically cleaner than $B \rightarrow K \pi$ but requires extremely large data samples. The current yield of $B^{-} \rightarrow D_{C P} K^{-}$events from BELLE is $\sim 16$ events in a $21 \mathrm{fb}^{-1}$ data sample [16]. This yield may be increased by about a factor of two by including additional CP eigenstates of the $D$ meson. Another approach is to use doubly Cabibbo suppressed decays (DCSD) of the $D$ meson. In this case, the event yield is much smaller but the direct CP asymmetries are much larger 15.

Measurement of a time dependent asymmetry in the mode $\bar{B}^{0} \rightarrow D^{*+} \pi^{-}$ also gives information on the angle $\phi_{3}$. In this case, however, the asymmetry is proportional to $\sin \left(2 \phi_{1}+\phi_{3}\right)$. The time dependence is also more complicated than in the case of a simple $\mathrm{CP}$ eigenstate such as $B^{0} \rightarrow \psi K_{s}$ [17]. To reconstruct $\bar{B}^{0} \rightarrow D^{*+} \pi^{-}$, one uses a partial reconstruction technique where the $\pi^{-}$and the low momentum pion from the $D^{*+} \rightarrow\left(D^{0}\right) \pi^{+}$decay are observed but the $D^{0}$ meson is not reconstructed. This increases the number of useable signal candidates by roughly an order of magnitude. Initial MC estimates indicate that, however, that even with the partial reconstruction method, $200 \mathrm{fb}^{-1}$ (which corresponds to 17000 lepton tagged signal events) will give a precision of \pm 0.34 for $\sin \left(2 \phi_{1}+\phi_{3}\right)$ in this channel[18].

For the methods described above one usually measures a trigonometric function of one of the angles such as $\sin \left(2 \phi_{1}\right)$. In such a case, there often exists multiple solutions or ambiguities for the angle itself (e.g. four solutions for $\phi_{1}$ from the initial $\sin \left(2 \phi_{1}\right)$ measurements). One cannot simply discard the solutions that are not consistent with the Standard Model. Instead to resolve these ambiguities, requires difficult high statistics measurements of quantities that depend on other functions of the angles such as $\cos (\phi)$. 
In addition to the program of measuring the angles of the unitarity triangle, there is also the question of whether there are new $\mathrm{CP}$ violating phases from new interactions or physics beyond the Standard Model. At the moment, such new phases are completely unconstrained. One way to attack this question is to measure the time dependent $\mathrm{CP}$ asymmetry in penguin modes such as $B^{0} \rightarrow \phi K_{s}$ or $B^{0} \rightarrow \eta^{\prime} K_{s}$ and compare it to the asymmetry in $B^{0} \rightarrow \psi K_{s}$. In the absence of new physics, they should be equal. However, if there are new physics contributions in penguin loops these asymmetries will differ substantially. Again an order of magnitude more data is needed for stringent tests [19, [20]. This search for new physics in CPV will be one of the most interesting aspects of the next phase in B factory physics.

\section{References}

[1] H. Albrecht et al. (ARGUS Collab.), Phys. Lett. B192, 245 (1987).

[2] A.B. Carter and A.I. Sanda, Phys. Rev. D23, 1567 (1981); I.I. Bigi and A.I. Sanda, Nucl. Phys. B193, 85 (1981).

[3] B. Aubert et al. (BABAR Collaboration), SLAC-PUB-8569, to appear in Nucl. Instrum. Methods.

[4] K. Abe et al. (Belle Collab.), The Belle Detector, KEK Report 20004 , to be published in Nucl. Instrum. Methods. and KEKB B-Factory Design Report, KEK Report 95-7.

[5] M. Kobayashi and T. Maskawa, Prog. Theor. Phys. 49, 652 (1973).

[6] T.E. Browder and K. Honscheid, Progress in Nuclear and Particle Physics, Vol. 35, ed. K. Faessler, p. 81-220 (1995).

[7] K. Abe et al. (Belle Collaboration), Phys. Rev. Lett. 87, 091802 (2001).

[8] B. Aubert et al. (BABAR Collaboration), Phys. Rev. Lett. 87, 091801 (2001).

[9] B. Aubert et al. (BABAR Collaboration), hep-ex/0107013, to appear in Phys. Rev. Lett. 
[10] K. Abe et al. (Belle Collaboration), Phys. Rev. Lett. 87, 101801 (2001).

[11] M. Beneke, G. Buchalla, M. Neubert and C.T. Sachradja, Nucl. Phys. B. 606,245 (2001).

[12] Y.Y. Keum, H.-N. Li, and A.I. Sanda, Phys. Rev. D 63, 054008 (2001).

[13] M. Ciuchini, E. Franco, G. Martinelli, M. Pierini and L. Silvestrini, Phys. Lett. B 515, 33 (2001).

[14] M. Suzuki, hep-ph/0001170.

[15] D. Atwood, I. Dunietz, and A. Soni, Phys. Rev. Lett. 78, 3257 (1997).

[16] K. Abe et al., BELLE-CONF-0108, paper submitted to the 2001 LeptonPhoton Conference.

[17] I. Dunietz, Phys. Lett. B. 427, 179 (1998).

[18] Y.H. Zheng (Belle Collaboration), private communication.

[19] For example, BABAR observes $10.8_{-3.3}^{+4.1} B^{0} \rightarrow \phi K_{s}$ events in 22.7 million $B \bar{B}$ events; B. Aubert et al., Phys. Rev. Lett 87, 151801 (2001).

[20] For example, BELLE observes $10.1_{-3.6}^{+4.4}$ and $6.4_{-2.7}^{+3.4} \eta^{\prime} K_{s}$ events in $\eta^{\prime} \rightarrow$ $\eta \pi^{+} \pi^{-}$and $\eta^{\prime} \rightarrow \rho \gamma$ modes; K. Abe et al., Phys. Lett. B. 517, 309 (2001). A sample of $11.1 \times 10^{6} B \bar{B}$ pairs is used. 

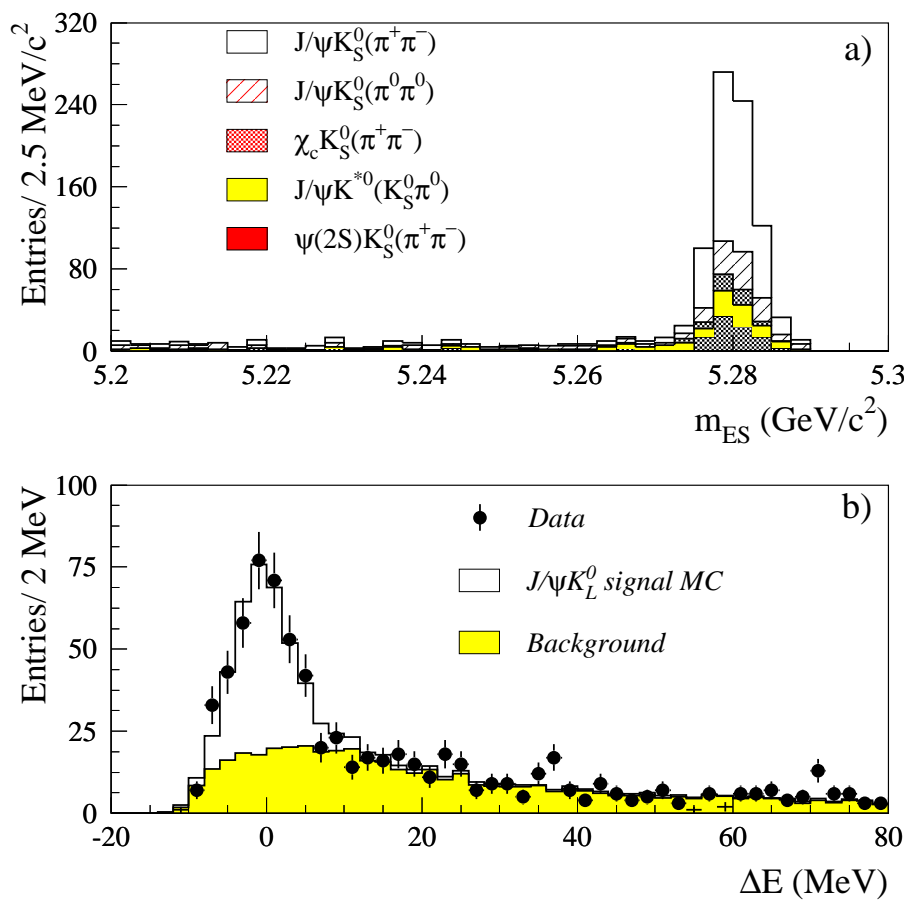

Figure 1: BABAR CP eigenstates. The upper figure shows the beam constrained mass distribution of the fully reconstrructed $\mathrm{CP}$ eigenstates. The lower figure shows the $p_{B}^{*}$ distribution for the $B^{0} \rightarrow \psi K_{L}$ candidates. 

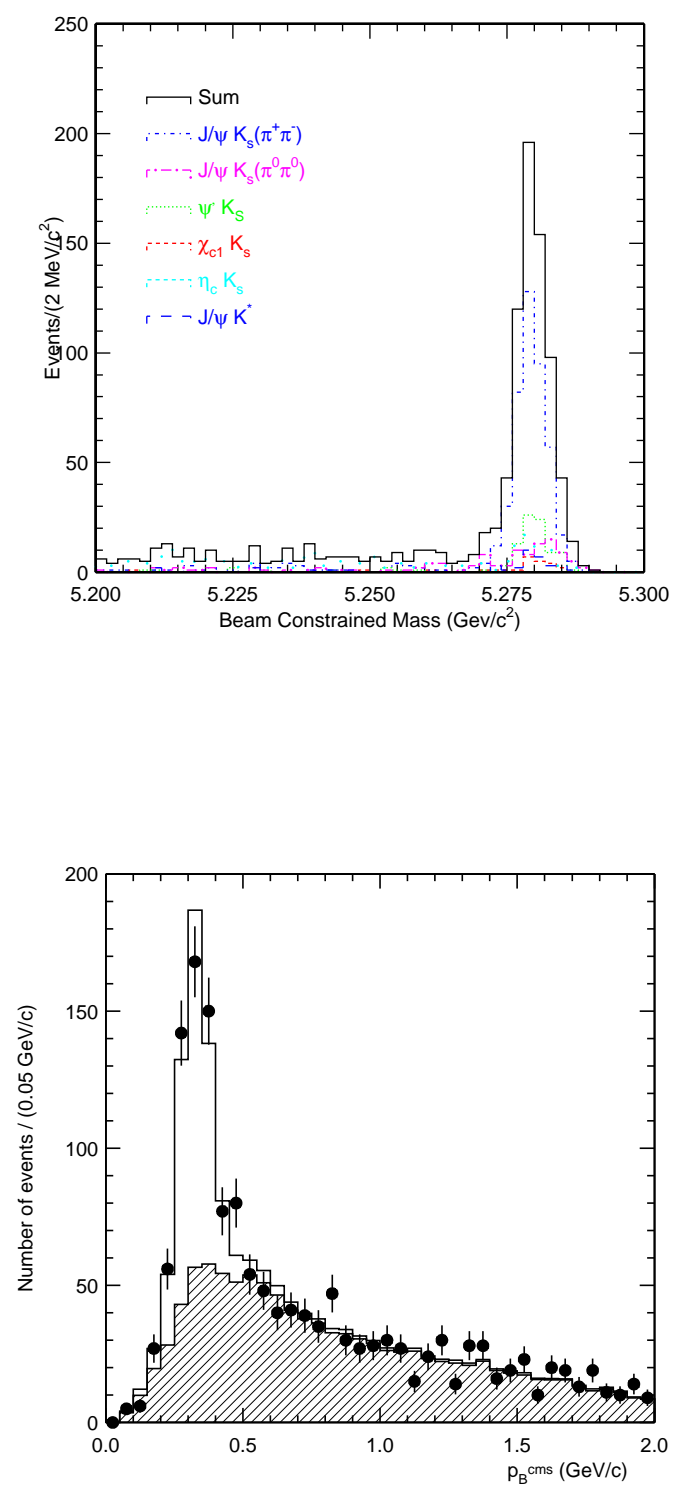

Figure 2: Belle CP eigenstates: the upper figure shows the beam constrained mass distributions for the exclusive modes while the lower figure shows the $p_{B}^{*}$ distribution for the $B^{0} \rightarrow \psi K_{L}$ candidates. 


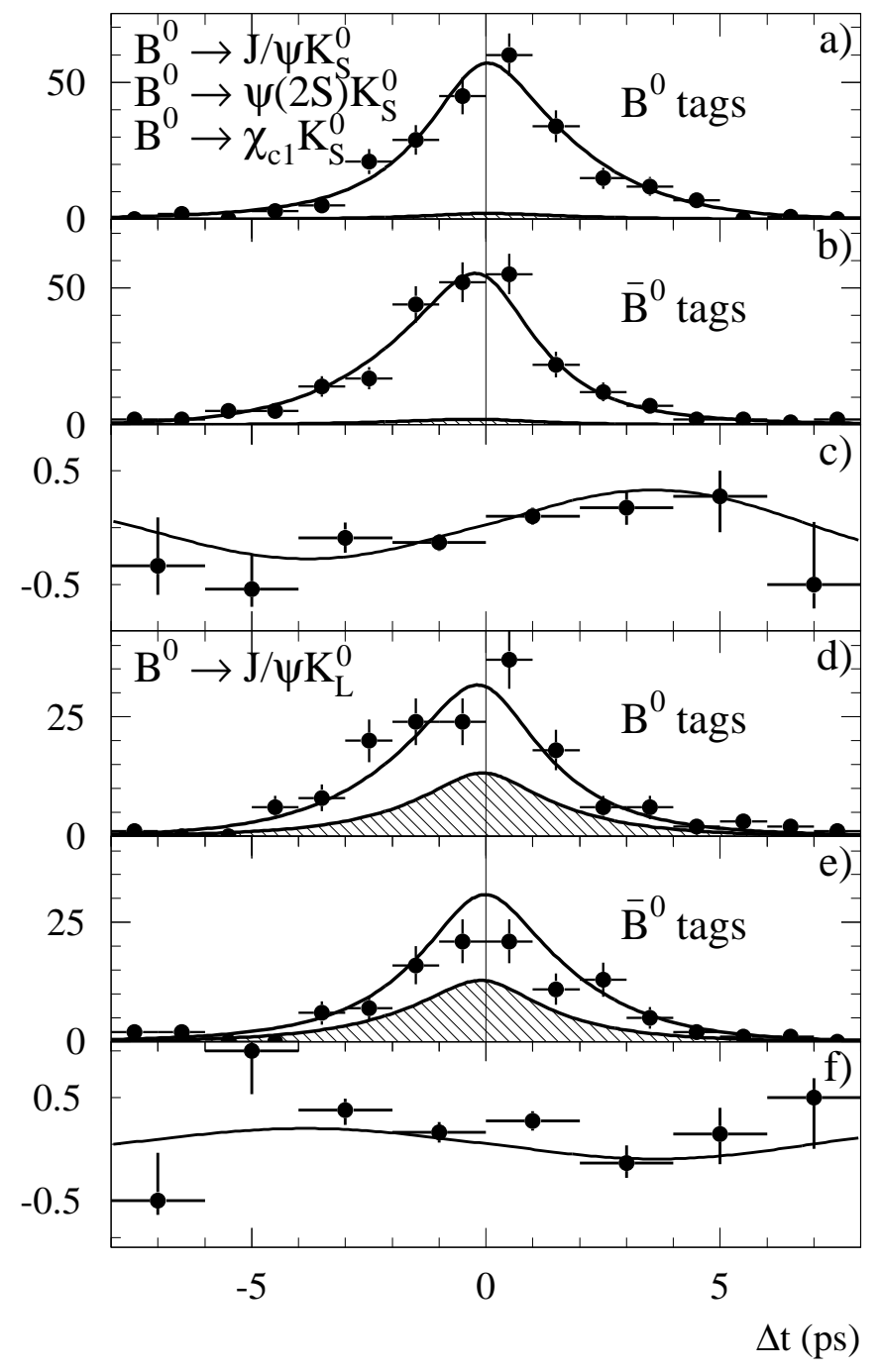

Figure 3: BABAR data on time dependent CP asymmetry. For the fully reconstructed $C P=-1$ final states, (a) The $\Delta t$ distributions for $B^{0}$ tags; (b) The $\Delta t$ distributions for $\bar{B}^{0}$ tags; the shaded area is the background, the solid curve is the fit to the data. (c) The time dependent asymmetry between (a) and (b); For the $\psi K_{L}$ final state, (d) The $\Delta t$ distributions for $B^{0}$ tags; (e) The $\Delta t$ distributions for $B^{0}$ tags; (f) The time dependent asymmetry between $(\mathrm{d})$ and $(\mathrm{e})$; 


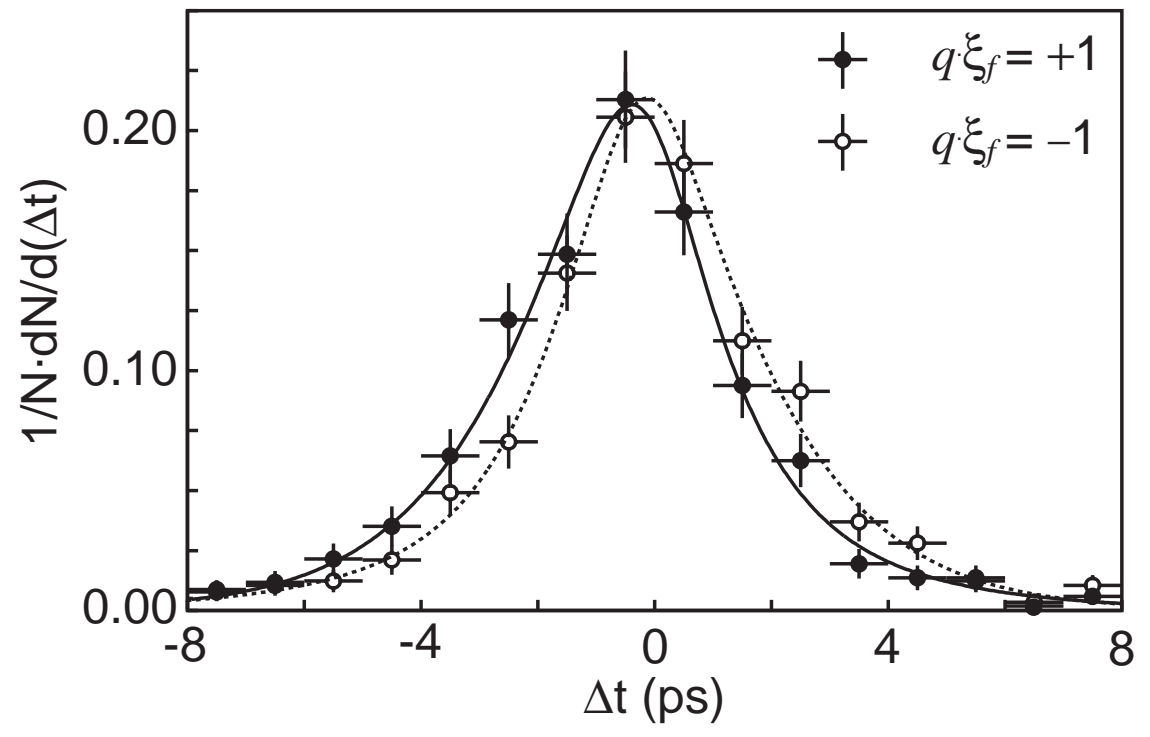

Figure 4: BELLE data on time dependent CP asymmetry. $\Delta t$ distributions for the events with $q \xi_{f}=+1$ (solid points) and $q \xi_{f}=-1$ (open points). The results of the global fit (with $\sin 2 \phi_{1}=0.99$ ) are shown as solid and dashed curves, respectively. 


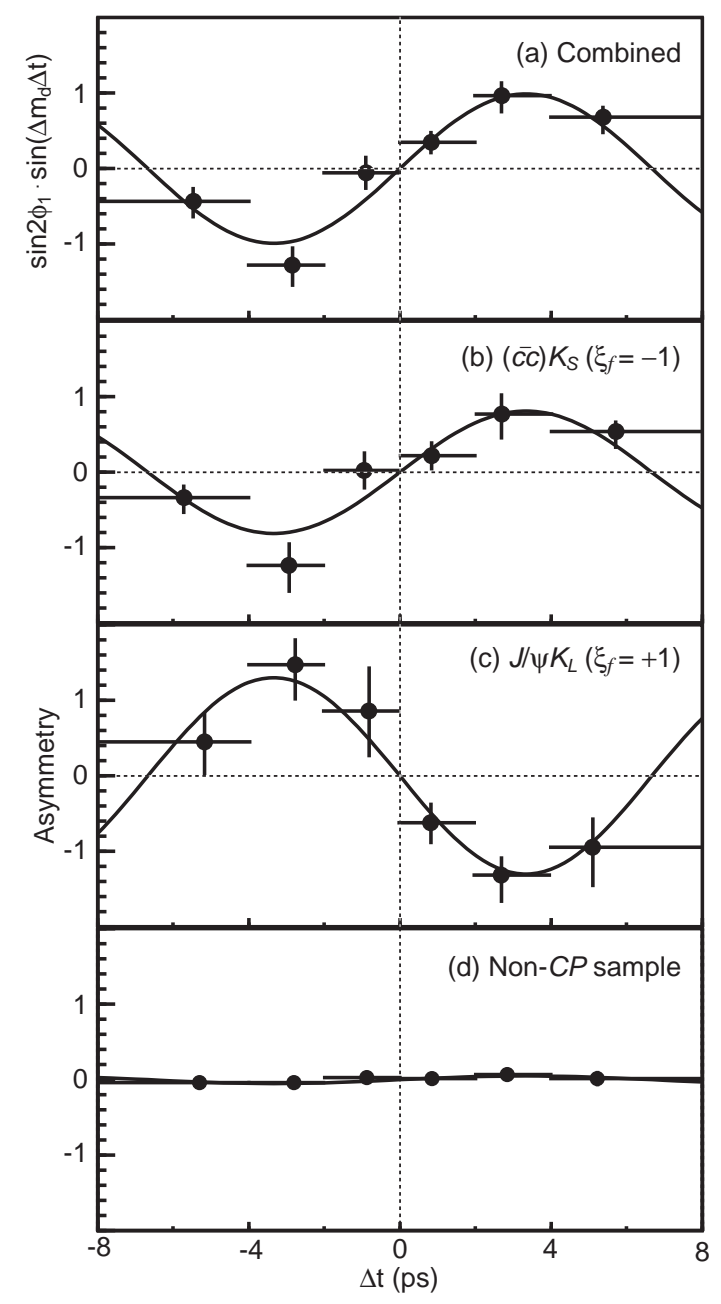

Figure 5: BELLE data on time dependent CP asymmetry. The raw $\Delta$ distributions for $B^{0}$ and $\bar{B}^{0}$ tags. (a) The asymmetry obtained from separate fits to each $\Delta t$ bin for the full data sample; the curve is the result of the global fit. The corresponding plots for the (b) $(c \bar{c}) K_{S}\left(\xi_{f}=-1\right)$, (c) $J / \psi K_{L}$ $\left(\xi_{f}=+1\right)$, and (d) $B^{0}$ control samples are also shown. The curves are the results of the fit applied separately to the individual data samples. 